LA-8463-MS

Informa! Report

(ISPO-110)

UC-15

Issued: July 1980

\title{
Irradiated Fuel Inspection in a Storage Pond with No Fuel Movement and an Uncollimated Detector
}

\author{
Phillip M. Rinard*
}

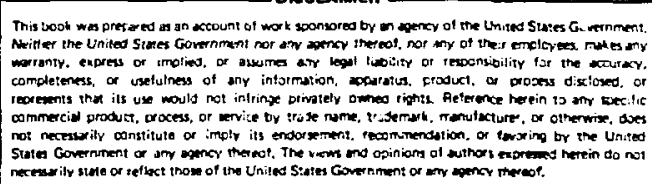

"Short-Term Visiting Staff Member. Physics Department, Emporia Stz̧e University, Emporia, KS 66801. 


\title{
IRRADIATED FUEL INSPECTION IN A STORAGE POND \\ WITH NO FUEL MOVEMENT AND AN UNCOLLIMATED DETECTOR
}

by

Phillip M. Rinard

\begin{abstract}
The practical limitations on the inspection of a large number of spent fuel assemblies in a storage pond make simple and rapid techniques a necessity. It is assumed here that the assemblies are not to be moved during the inspection in order to reduce the time required. The task is simplified further when the detector is well collimated. If the collimation is insufficient to respond to only one assembly from among an array of fuel asserblies, then an unfolding process is needed and that is the subject of this report.

A formulation of the detector's behavior is given followed by an unfolding process to yield the detector's response to each assembly as if it were isolated. Some simulated arrays are used to illustrate the sensitivity of the procedure to normal fluctuations in the measured quantities. Measurements with an ionization chamber on actual arrays of up to fourteen PWR assemblies are treated to pro$v$ ide a realistic application of the unfolding process.

An unfolding technique is, of course, essential with a poorly collimated detector, but the examples warn that weak source strengths surrounded by much stronger sources will not be accurately recovered and even the detection of voids is thus uncertain. Some simulated arrays of up to 100 assemblies indicate that unacceptably inaccurate results may be caused by even the small normal fluctuations in measured values. Any use of an unfolding process is thus quite limited.
\end{abstract}

\section{INTRODUCTION}

The ability to estimate the burnup for each spent-fuel assembly in a storage pond without any assembly movement would greatly speed an inspection and allow a more comprehensive inspection during an available time period. A 
detector that gives a single numerical response in a short amount of time would also contribute to a more rapid inspection. These are consistent with the IAEA desire "that the number, intensity, duration, and timing of routine inspections shall be kept to the minimum consistent with the effective implementation of the safeguards procedures...." In the analysis that follows, it is assumed that such a simple detector is held above each individual fuel assembly. With more thian one assembly affecting the detector at a time, an unfolding process is needed to determine the response due to each individual assembly.

The responses must eventually be correlated with the burnup values. Some features of this process and its problems are given in Appendix $A$.

While some of the exarnples discussed here deal with a gross-gamma detector (e.g., ion chamber), the unfolding procedure does not depend on the type of radiation or detector. The radiation could be gamma rays, neutrons, or light, for example, and the detector could respond to all the radiation or have thresholds or discriminators. If a detector is always affected by only one asseribly at a time due to collimation, then the unfolding process is not needed.

\section{UNFOLDING PROCEDURES}

\section{A. Analytical Process}

For assemblies with wide ranges of activities and for detector positions restricted by the assembly superstructure and rack structure, it may not be possible to have the perfect collimation. In general, the dose rate in a detector is the sum of dose rates from many assemblies. It is assumed here that separate dose rates add linearly in the detector to create the detector's total response. While interference of the radiations from adjacent assenblies should not be a problem, a real detector may saturate or not respond linearly. For a nonlinear detector, the unfolding can still be reduced to solving a set of linear equations (Appendix B), and the procedures described here would then apply to that case also.

If a detector is placed above the assembly in Fig. I with coordinates $\left(x_{0}, y_{0}\right)$, the detector response $D_{x_{0} y_{0}}$ found is

$$
\sum_{x} \sum_{y} c_{x y}^{x_{0} y_{0}} d_{x y}=D_{x_{0} y_{0}},
$$




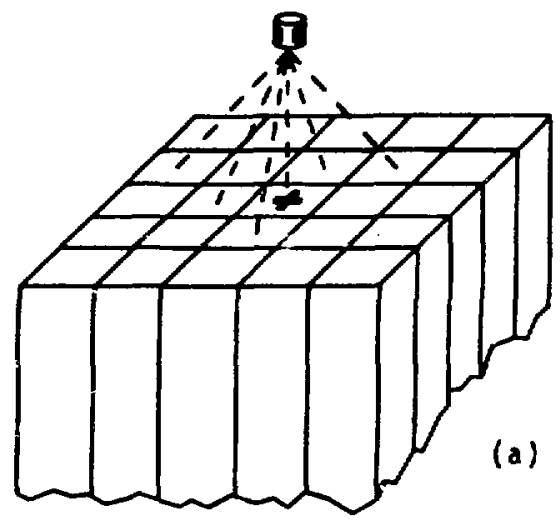

Fig. 1 .

(a) A detector is located above one assembly in a storage rack, but radiations from many assemblies reach it.

(b) As seen from above the rack, each square represents one assembly (or a void). The detector is at $x=7$ and $y=5$ assembly widt' units from the lower-left corner of the rack. This style of drawing will be continued in many of the other figures.

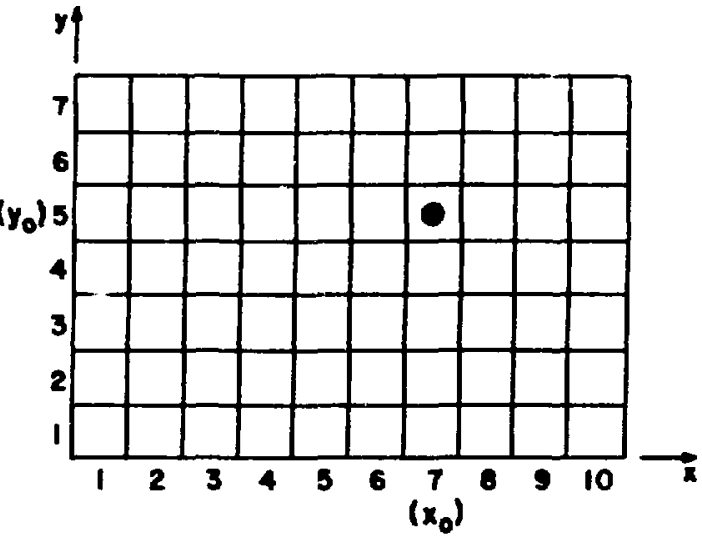

(b)

where $d_{x y}$ is a detector response from directly above an assembly at $(x, y)$ when it is isolated from all other assemblies and the $c_{x y}^{x_{0} y_{0}}$ coefficient is the fraction of $d_{x y}$ found at a location $\left(x_{0}, y_{0}\right)$ which is some distance from the overhead position $(x, y)$. For an isolated assembly, $c_{x y}^{x_{0} y_{0}}=0_{x_{0} y_{0}} / d_{x y^{*}}$. When the detector is overhead, $(x, y)=\left(x_{0} y_{0}\right)$ and $C=1$. The sums on $x$ and $y$ in Eq. (1) range over all the assemblies that significantly contribute to the detector response for a given $\left(x_{0} y_{0}\right)$. The Cs and d's also depend on the height above the assemblies, but if this is held constant it need not be shown explicitly.

It is indicated in Appendix $A$ how the $d_{x y}$ might depend on burnup, cooling time, and irradiation history. (The cs might also depend on these parameters.) For a given gamma-emitting assembly in water, the relative dose rates at two positions will depend on the mixture of the gamma rays with different energies. If high energies dominate, for example, then the Cs will vary more slowly with distance. 
Since the $D_{x_{0} y_{0}}$ are measured, and the $d_{x y}$ are to be found, it is necessary to know the Cs accurately. In principle, they can be calculated, given sufficient understanding of spent fuel assemblies. In practice, it may be best to try to develop semi-empirical estimates for given sets of irradiation history. In any case, for noninterfering radiation and a linear detector, Eq. (1) applies and is a set of linear algebraic equations for the $d_{x y}$

It is convenient to treat this equation as a multiplication of the unknown d's, taken as a column vector, by a matrix of the Cs.

$$
[c][d]=[0] \text {. }
$$

If all the rack locations are used, then the C-matrix will iave a zero column for each void in the rack. To simplify Eq. (2) when a void exists at $(x, y)$, column $(x, y)$ and row $(x, y)$ of the C-matrix are excluded, as are the $(x, y)$ components of the $d$ and $D$ vectors. In this manner, a 10 by 10 rack with only 16 assemblies present in it entails only a 16 by 16 matrix instead of a 100 by 100 matrix.

\section{B. Computational Process}

There are several ways to find the d's from Eq. (2). ${ }^{2,3}$ While they are equivalent in principle, the characteristics of a computer tend to favor one numerical method from the others. The method that uses the fewest number of arithmetic operations and thereby minimizes difficulties from round-off errors is Cholesky's method (also called Crout's method, the method of matrix decomposition, and the method of matrix factorization). ${ }^{2}$ It is also economical in regard to memory storage locations since the auxiliary matrix elements required can be stored in locations within the C-matrix itself.

Two improvements $c$ an be made on this basic procedure. The diagonal elements of the C-matrix are used as divisors in Cholesky's method and if one of them is much smaller than the other elements, the solution's accuracy can suffer. This can be avoided by interchanging rows until the diagonal elements are all as large as possible (the "partial pivoting" process ${ }^{2}$ ). In the case of spentfuel inspection, the diagonal elements of $C$ are all 1.0 and the off-diagonal elements are mast likely less than 1.0 , so this improvement is not needed. 
However, since the C-matrix is $N$ by $N$, where $N$ is the number of assemblies, it is seen that a full storage pond will lead to a very large matrix. In a square rack, only ten assemblies on a side, there are 100 assemblies and therefore 100 simultaneous equations. With as few as 20 equations to solve, accuracy can be lost in the seventh figure. ${ }^{2}$ So one must proceed with caution when so many assemblies are examined. The improvement for this case is the so-called error equation. ${ }^{2}$ The solution vector $d$ from Cholesky's method is put back into Eq. (2) and multiplied by the C-matrix, yielding a new vector $D^{\prime}$. If $D^{\prime}$ is sufficiently similar to the known $D$, nothing more needs to be done; the solution is accurate. If errors have entered into $d$, however, the $D-D^{\prime}$ is not close to zero. By replacing $d$ with $d+\Delta d$ and $D$ by $D^{\prime}-D^{\prime}$ in Eq. (2), a new set of equations for $\Delta d$ is solved.

$$
[C][\Delta d]=\left[D-D^{\prime}\right]
$$

If $d+\Delta d$ still contains unacceptable errors, the process can be iterated until the corrections are ignorable. Little additional memory is required to do this since the $\mathrm{C}$-matrix can be regenerated for each iteration to replace the old $C$-matrix destroyed by the Cholesky method; a second $C$-matrix need not be stored in memory for use at the beginning of each iteration.

Since three- or four-figure accuracy is adequate in this spent-fuel analysis, it has been found that at least 100 assemblies can be treated without requiring the error equation. It was seen that exceptions occur (even for nine assemblies) when some d's differ by factors of many thousands. Since the d's will not generally differ by such large factors in practice, the error equation will rarely be needed. To solve 100 equations on a PDP $11 / 60$ minicomputer takes only eight seconds, so computational time need not be a problem.

\section{EXAMPLES}

\section{A. Simple Example}

This example will illustrate how to construct the C-matrix. Figure 2(a) shows a rectangular ( 2 by 3 ) array of four assemblies and two voids. It is assumed for simplicity that a detector will provide a measurable response from 


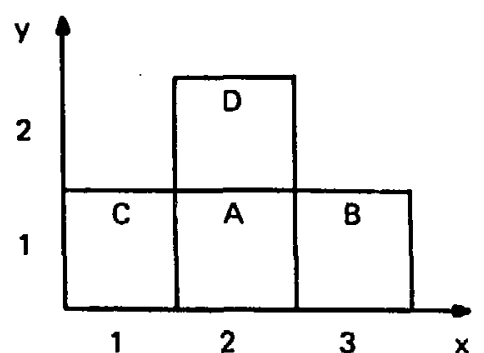

(a)

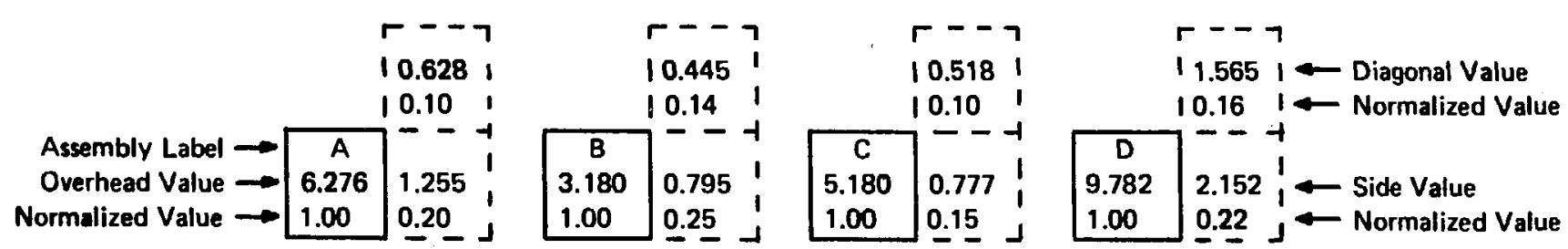

(b)

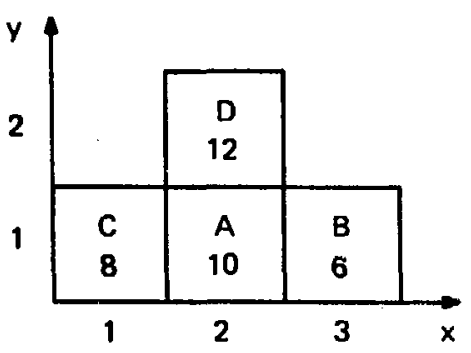

(c)

Fig. 2 .

An arrangement of four assemblies is in (a). Each has a different set of Cs, and they are given in (b) below an identification symbol and some assigned "data" from the assemblies when isolated. (For example, directly above $A$ gives a detector value of 6.276 , while one position to the side of $B$ gives 0.795. ) These data combine to produce the detestor's responses $D$ in $(c)$. Unfolding these responses returns the overhead values of $d$ shown in (b). 
an isolated assembly only when it is (a) directly above the assembly, (b) one rack position off to the side, or (c) one rack position away along a diagonal. These types of positions are shown in fig. 2(b). Each portion of Fig. 2(b) shows an isolated assembly and the detector's readings at the three types of positions. The $C$ values are given on the bottom of the boxes and are simply the readings divider by the overhead value.

When the detector is placed above an assembly in Fig. 2(a), there will be a sum of contributions recorded, as in Eq. (1). These are shown in Fig. 2(c) and are the data $D$.

To build the C-matrix in this case, use the Cs from Fig. 2(b) and first consider the contributions to the detector with $\left(x_{0}, y_{0}\right)=(1,1)$. Assemblies $C$, $A$, and $E$ contribute $1.0,0.2$, and 0.16 for the $C_{11}^{91}, C_{21}^{11}$, and $C_{22}^{11}$, respectively. The $C_{31}^{11}$ is zero since assembly $B$ is too far from $C$ to affect the detector. These are placed in the first row of the C-matrix.

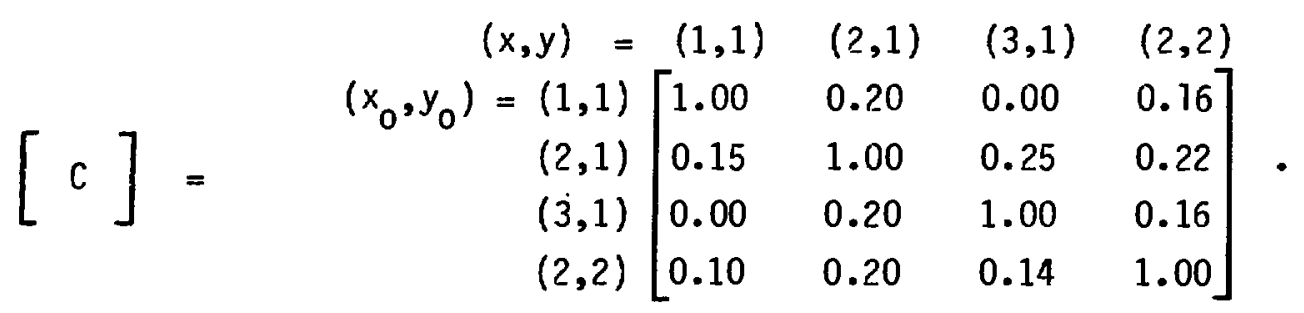

Moving the detector above $A$ at $(2,1)$ gives the second row, and so on. Notice that a vacant location in the storage rack is ignored in the C-matrix.

The activities of the individual assemblies can now be recovered using the matrix of Eq. (4) and the "data" of Fig. 2(c). Naturally, the d's found are those shown in Fig. 2(b) since they were used to create the "data" of Fig. 2(c) in the first place. An obvious difficulcy in a real situation is to confidently know all the Cs without isolating every assembly, thereby violating the original intent of not moving the fuel. The Cs may depend on the irradiation history, the cooling time, and the detector's distance above the assembly. It will be seen later that, in practice, one general set of Cs can be determined and used for assemblies with wide ranges of values of these parameters. 


\section{B. Sensitivity to Errors}

To examine the sensitivity of the deduced Ds to errors in the Cs, the values of the CS in Fig. 2(b) were altered for positions not directly above an assembly. The d's were recalculated and compared to the correct ones in Fig. 2(b). It was found that 10 percent errors in the cs led to errors in the d's of less than 5 percent. However, 50 to 100 percent errors in the Cs gave $d$ values also in error by 50 to 100 percent. Since small uncertainties in the Cs give even smaller uncertainties in the Ds for this little array, there is encouragement that the process may be useful.

Another set of errors will arise in the measured Ds. To investigate their importance, the correct $C s$ shown in Fig. 2(b) were used while the Ds in Fig. 2 (c) were modified. The new d's found were again compared to those in Fig. 2(b). A 10 percent error in a $D$ gave about a 20 percent error in the new d, indicating that the results are much more sensitive to errors in the data from a full rack than from isolated assemblies.

To examine these matters further, a 10 by 10 array was created using four types of assemblies. Each type was given a unique set of the $C s$ and $a d$. These were used to calculate the correct detector response (the Ds) above each of the 100 assemblies.

After changing some of the Cs by 3 percent or less, new d's were computed and compared to the correct values. Even such small changes in the Cs caused many of new d's to be incorrect by more than 30 percent, and some were even negative. Similar effects were found after changes in the simulated detector responses, the Ds.

This case of a large array was clearly more sensitive to small errors in the "data" than was the earlier small array example.

\section{Subdividing a Storage Rack}

If a storage rack has dimensions of, say, 40 by 30 assembly-size units, there are up to 1200 assemblies present. This could lead to a 1200 by 1200 C-matrix and 1200 equations to solve simultaneously. While not a problem in principle, there are some practical matters of importance in a case like this. It may be unlikely that an IAEA inspector would want to map a complete rack even if he had the hours available in which to do it. Secondly, a computer would need a memory of more than 1440000 words to do the task. The time 
required to solve Eq. (2) may be only a few minutes and even assuming the error equation is needef the total computer time would still be a very small fraction of the data acquisition time.

If the detector is so well collimated that it is affected by only one assembly at a time, then all of this is unnecessary. But assiming, as has been done earlier, that continguous assemblies also contribute to the detector's output, what can be done to avoid working with a complete rack

Perhaps one could measure above an assembly and a surrounding subset of the entire storage rack. Using this subset as if it were an isolated rack, the results for assemblies on the apparent boundary would surely be in great error (unless they happened to also be part of the original, true boundary). But perhaps the error would be less for assemblies closer to the center, until finally a nearly correct value would be obtained for the one of interest. If the subset were only 5 by 5 or 7 by 7 , an individual assembly in a large rack could be studied without the massive effort needed for the complete rack.

To investigate this possibility, subsets of the 10 by 10 rack mentioned in the previous section were treated as complete racks. With position $(6,6)$ at the centers, arrays ranging in size from 3 by 3 to 9 by 9 were chosen and the d's found. Additionally, 5 by 5 subsets at the corners and sides of the 10 by 10 rack were selected and the d's again found.

These results give little encouragement to use subsets in this manner. Even with the 9 by 9 there are few results within 10 percent of the correct values and the central assemblies are hardly more accurate than those on the boundaries. Only in examining assemblies very near actual corners is there much promise, but it would be less work to physically isolate those few and avoid the unfolding process.

From a purely mathematical standpoint, this failure to unfoid only a portion of an array is reasonable since it amounts to attempting to solve only a portion of Eq. (1). If there are ten colspled equations, then four of the equations and four unknowns can not simply be ignored, for exarnple.

Subsections of a rack can be created so that they are truly isolated, however. If only assemblies contiguous to the one directly below the detector can affect the detector's readings (as has been assumed so far), then a vacant row or column is sufficient to form independent subsections. For a 10 by 10 rack, if row 4 were empty, then the lower 10 by 3 section could be treated as if the upper 10 by 6 did not exist, and vice versa. 
In reality, it would doubtless be necessary to move assemblies to create such vacant boundaries. If only one assembly was of interest, then the simplest movement is to take it to an isolated spot by itself. To study all the assemblies within an $n$ by $n$ square subsection, it. is easiest to isolate them one-by-one if $n$ is less than 5 . For larger subsections, less fuel movement would be dons by vacating the positions bordering the $n$ by $n$ subsection and solving the $n^{2}$ equations.

If two consecutive vacant rows or columns were needed to isolate sections from each other, then the smallest subsection where creating an isolation boundary would require less fue movement is a rather large 10 by 10 array.

\section{Locating Simple Inhomogeneities}

It could be possible to locate one assembly out of many without resorting to an unfolding process if all but that one are fairly uniform in source strength. For the case when each assembly produces responses of 10,3 , and 1 above, laterally, and diagonally beside the assembly, then the detector response above every assembly (except those on the border) will be 26 . If one assembly in the middle of these now has a source strength only 80 percent of the others, the detector above it will give 24 ; this is 92.3 percent $r{ }^{11}$ the others. If an assembly is completely removed, the reading above the void is 16 , or 61 percent of the others.

If each assembly gives detector readings of 10,2 , and 1 (collimation is improved), then the same two inhomogeneities yield 01 percent and 55 percent of all the other locations. This slightly better collimation naturally makes the irregularities more conspicuous.

The contributions from neighboring assemblies obviously tend to smooth the detector's readings over irregularities and make it more difficult to detect inhomogeneities without an unfolding operation.

\section{EXPERIENCE WITH PWR ASSEMBLIES}

To examine the unfolding process in a real situation, fourteen assemblies at a PWR nuclear power plant were measured in various combinations and configurations. An ionization chamber at the hottom of a tube was positioned above an assembly with the aid of horizontal cross members to fix the vertical height and to assist in aligning the chamber above the center, of the assembly. ${ }^{4}$ It 
was found that the detector could be moved horizontally off the axis as much as $5 \mathrm{~cm}$ without significantly changing the reading. Positioning errors should thus have no consequence.

Figure 3 shows three regions of a normally empty portion of the storage rack. This is seven rows away from the nearest stored irradiated assembly. From the 4 by 4 initial section, assemblies were noved to the final left-hand triangular region to construct different arrays; by doing this, new arrays were automatically produced in the 4 by 4 section also. The middle section was used to isolate an assembly temporarily while measurements were taken above and near it; these data gave the $d$ and $C s$ for that assembly.

\section{A. The Measured C Coefficients}

The available ranges of burrup and cooling time values are shown in Fig. 4; the assemblies selected for use are labeled near their correct values. Six of them, one from each of the six general regions in Fig. 4, were isolated and their Cs determined. All assemblies with similar burnup and cooling time were assumed to have the same $C S$ so that only these six would actually have to be examined in detail. The data and the resultant Cs are shown in Fig. 5. The ionization chamber display was set to read zero with no assembly near it and no consistent background value is apparent in Fig. 5. (The calculated d's found later were also best when no further background subtraction was made.)

FINAL

ISOLATION

INITIAL

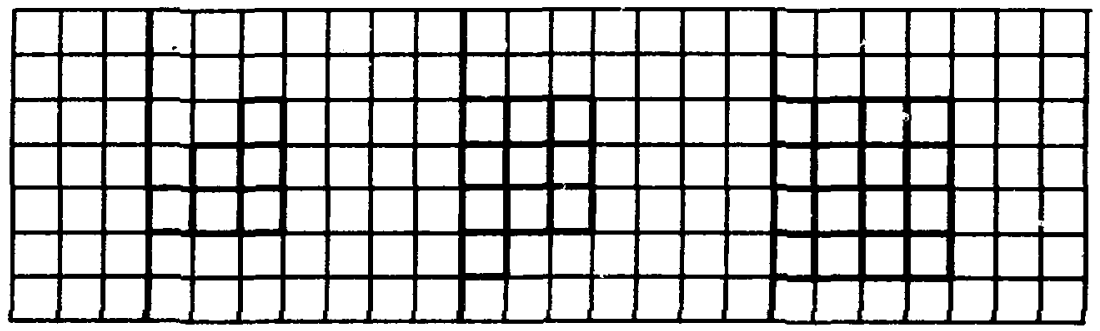

Fig. 3 .

This is a portion of the PWR storage rack that normally contains no assemblies. The nearest irradiated assembly is seven rows away. Fourteen assemblies were moved to the 4 by 4 array labeled "INITIAL." Some were examined in the "ISOLATION" section to measure their CS; all were isolated to measure their d's. Arrays were also formed in the "FINAL" section from some of the fourteen assemblies. 


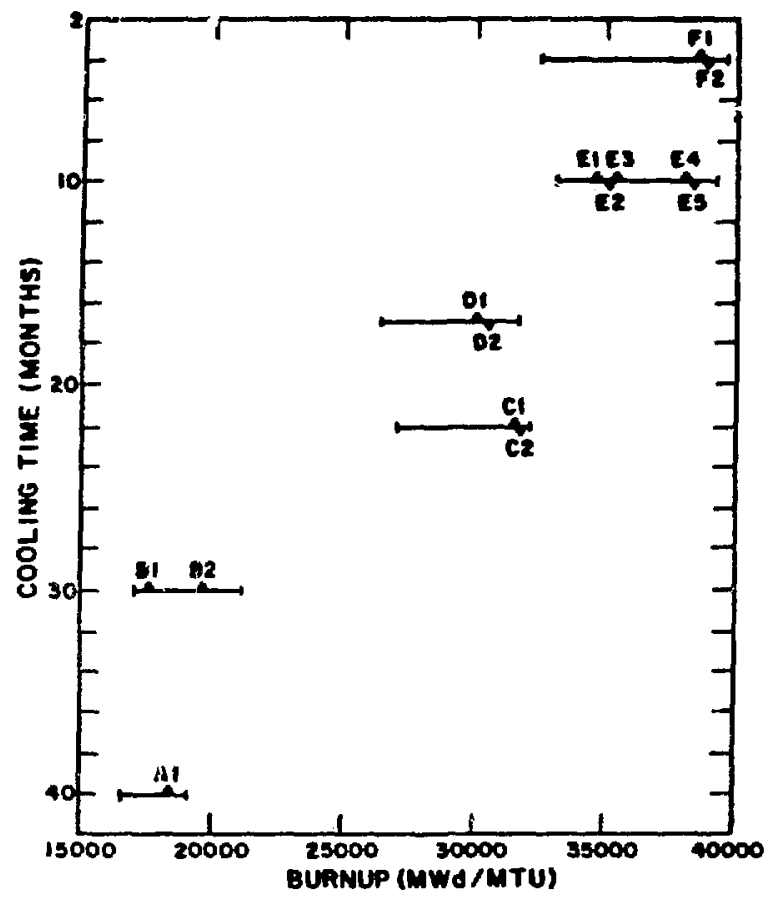

Fig. 4.

The fourteen PWR assemblies used in the rack of Fig. 3 to create small arrays had the burnup and cooling time values shown here. The combinations arailable in the whole pond are along the six horizontal lines. The labeled marks show the assemblies selected.

These same data and CS are shown graphically in Fig. 6. It is most apparent in Fig. 6(b) that for the two lowest burnup assemblies (which also had the longest cooling time), the data do not simply decrease with distance. This is possibly due to difficulties in measuring these very low values (see Fig. 5) or to contamination on the storage rack. In any case, this behavior is not taken to be the true situation.

it is also apparent in Fig. 6(b) that whatever curves are drawn through the points for the four tigher burnup assemblies, they will not diffar very much despite their wide range of burnup and cooling times. Some previous work $^{5}$ with assemblies from this same PWR plant suggests why this should be so. The percent of the dose from each particular energy gamma ray in an airfilled chamber near the assemsities was found to be nearly independent of the burnup and cooling time. In this case, it is reasonable that the Cs are also independent of the assembly since they are measured under the same conditions as existed in the calculations of Ref. 5 .

Another question that can be raised about the Cs is whether they are the same for an assembly when isolated and when within an array. If the detector is not directly above an assembly, radiation from the assembly's top and side could both affect the response. Within an array, however, a neighboring assembly might shield the detector from that radiation emitted from the side and the Cs obtained for an isolated assembly would not apply. 


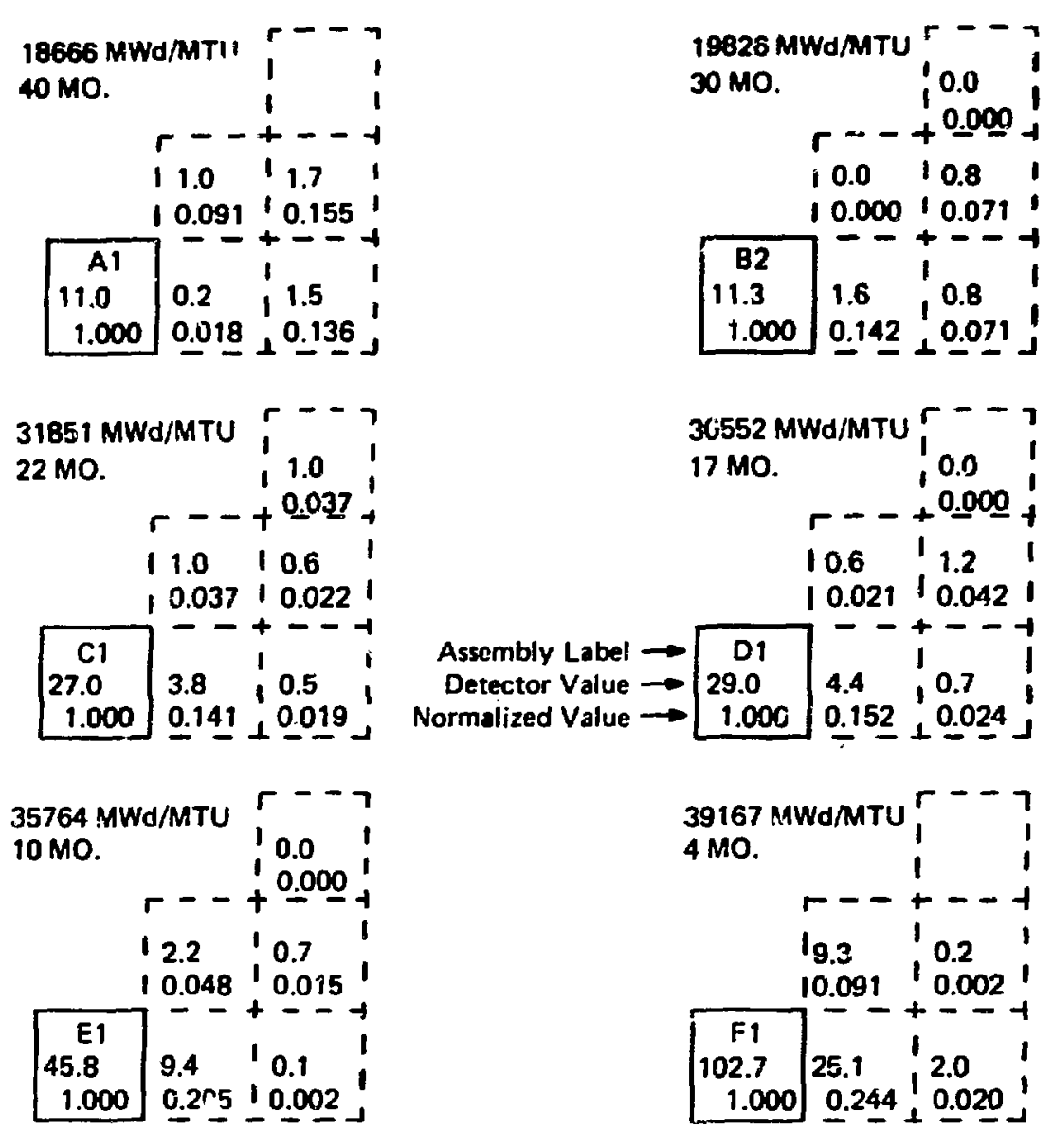

Fig. 5 .

Six PWR assemblies were studied in detail, one from each of the regions in Fig. 4. The data and derived CS are given in the boxes.

Two experimental arrays strongly indicate that this is not the case. In a linear array of three assemblies, the center one had a very low burnup and a long cooling time. Its removal made no difference to the measurements above the other two. This shows that the radiation reaching the detector must come from the uppermost portion of the assembiies that cannot be shielded by a neighbor. In an 4 hy 4 array, the removal of a low burnup assembly again had no affect on its neighbors. Other studies ${ }^{5}$ have demonstrated that indeed very little radiation from the side reaches any overhead location, so it is not surprising to find these results with a detector above the assemblies. 


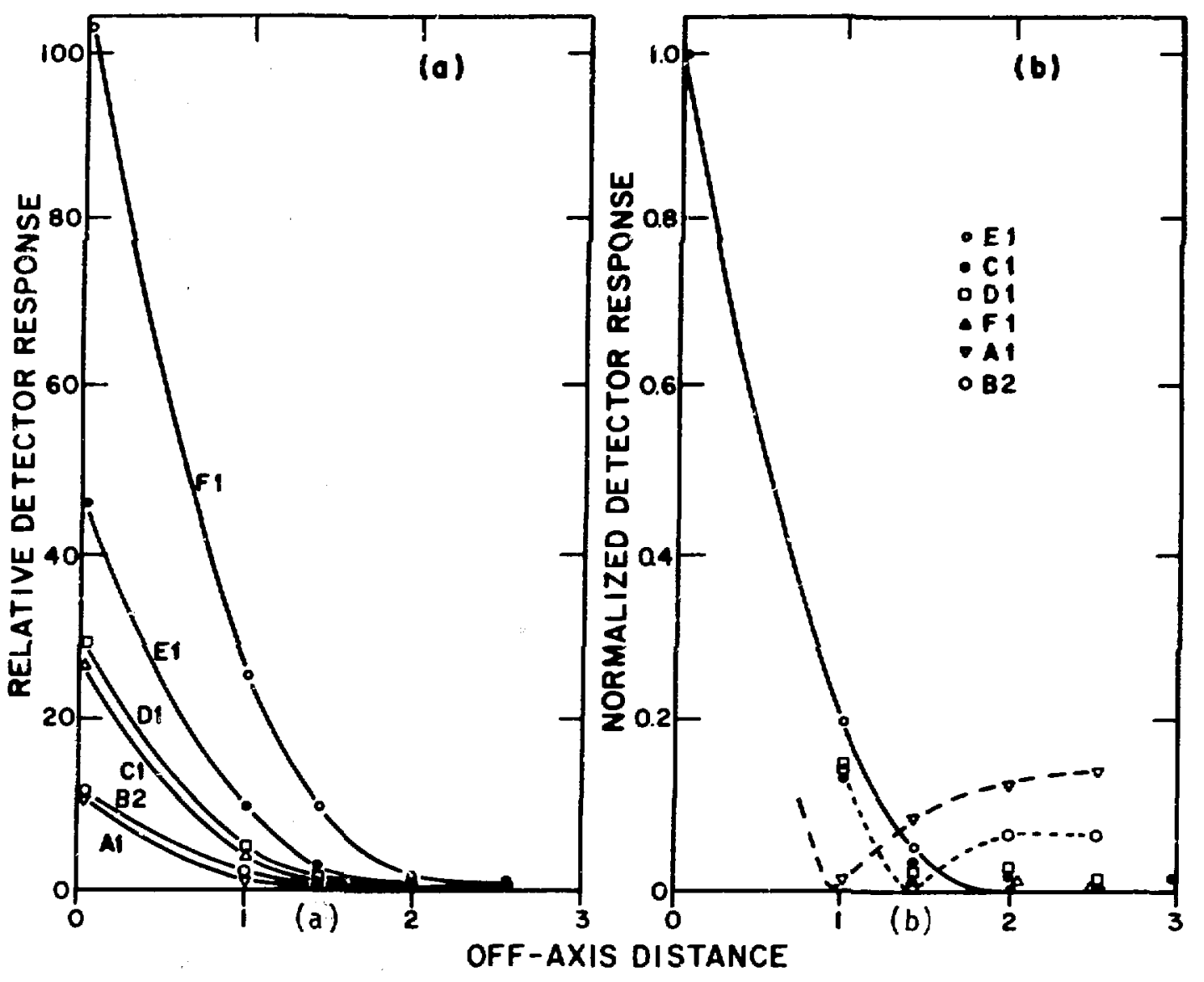

Fig. 6 .

The data and CS of Fig 5 are plotted here. In (a) the relative sizes of the Cs among the assemblies are shown, while in (b) they have all been normalized to one at the overhead position. The off-axis distance is $\sqrt{[}\left(x-x_{0}\right)^{2}+$ $\left.\left(y-y_{0}\right)^{2}\right]$. The solid curve in (b) was eventually chosen as the best smoothed values for the $C s$.

\section{B. The Measured d Source Activities}

The "correct" d's are shown in Table I in the cciumin labeled by "I", which means that each assembly was measured from directly above when isolated. The assemblies are grouped by burnup and cooling time. These d's are usually the result of a single measurement, but where more than one was taken, it was clear that the third significant figure had little significance. In any case, these d's must beconse the standards of comparison for the unfolded d's obtained in more complicated arrays. 
TABLE I

SOLUTION SUMMARY*

\begin{tabular}{|c|c|c|c|c|c|c|c|c|c|c|c|c|c|c|c|c|c|}
\hline $\begin{array}{l}\text { Nd/MTU } \\
\text { (months) }\end{array}$ & ID & 1 & $2 a$ & $2 b$ & $2 c$ & $2 d$ & $3 a$ & $3 b$ & 4 & 5 & $6 a$ & $6 b$ & 8 & 10 & 12 & 13 & 14 \\
\hline $\begin{array}{c}18000 \\
40\end{array}$ & A1 & 11.0 & & & & 8.1 & & 6.1 & & & & 5.3 & & 7.8 & 10.0 & 2.2 & 0.8 \\
\hline $\begin{array}{c}19 \quad 00 \\
30\end{array}$ & $\begin{array}{l}B 2 \\
B 1\end{array}$ & $\begin{array}{r}11.3 \\
8.0\end{array}$ & & & & & & & & & & & $\begin{array}{r}11.1 \\
1.9\end{array}$ & $\begin{array}{r}11.1 \\
2.5\end{array}$ & $\begin{array}{l}9.8 \\
8.0\end{array}$ & 3.9 & $\begin{array}{l}9.0 \\
2.7\end{array}$ \\
\hline $\begin{array}{c}32000 \\
22\end{array}$ & $\begin{array}{l}\mathrm{Cl} \\
\mathrm{C} 2\end{array}$ & $\begin{array}{l}27.0 \\
26.0\end{array}$ & $\begin{array}{l}27.6 \\
28.4\end{array}$ & $\begin{array}{l}27.0 \\
27.7\end{array}$ & 29.8 & & $\begin{array}{l}26.8 \\
27.1\end{array}$ & 29.6 & $\begin{array}{l}27.1 \\
24.0\end{array}$ & $\begin{array}{l}29.3 \\
25.3\end{array}$ & $\begin{array}{l}26.6 \\
27.1\end{array}$ & $\begin{array}{l}28.4 \\
25.0\end{array}$ & & & 30.7 & $\begin{array}{l}27.8 \\
33.5\end{array}$ & $\begin{array}{l}29.1 \\
30.8\end{array}$ \\
\hline $\begin{array}{c}31000 \\
17\end{array}$ & $\begin{array}{l}02 \\
D 1\end{array}$ & $\begin{array}{l}37.1 \\
29.0\end{array}$ & & & & & & & & & 24.0 & & $\begin{array}{l}35.9 \\
33.4\end{array}$ & $\begin{array}{l}36.3 \\
33.4\end{array}$ & $\begin{array}{l}40.0 \\
27.7\end{array}$ & $\begin{array}{l}37.5 \\
30.7\end{array}$ & $\begin{array}{l}36.5 \\
31.6\end{array}$ \\
\hline $\begin{array}{c}36000 \\
10\end{array}$ & $\begin{array}{l}E 4 \\
E 1 \\
E 5 \\
E 2 \\
E 3\end{array}$ & $\begin{array}{l}48.1 \\
46.8 \\
38.4 \\
42.1 \\
42.6\end{array}$ & & & & & 45.5 & & $\begin{array}{l}48.7 \\
47.2\end{array}$ & $\begin{array}{l}51.4 \\
48.3\end{array}$ & $\begin{array}{l}52.1 \\
46.8\end{array}$ & $\begin{array}{l}49.3 \\
45.2\end{array}$ & $\begin{array}{l}42.5 \\
39.8 \\
42.5\end{array}$ & $\begin{array}{l}42.5 \\
39.8 \\
42.5\end{array}$ & $\begin{array}{l}50.0 \\
48.6 \\
40.9 \\
45.3 \\
40.4\end{array}$ & $\begin{array}{l}57.2 \\
51.8 \\
40.0 \\
46.8 \\
44.9\end{array}$ & $\begin{array}{l}56.5 \\
50.0 \\
42.6 \\
46.5 \\
41.9\end{array}$ \\
\hline $\begin{array}{c}39000 \\
4\end{array}$ & $\begin{array}{l}F 1 \\
F 2\end{array}$ & $\begin{array}{l}145.7 \\
102.0\end{array}$ & & & 100.0 & 144.0 & & 101.0 & & 104.0 & 111.0 & 102.0 & 103.0 & $\begin{array}{l}142.0 \\
100.1\end{array} \mid$ & 144.0 & $\begin{array}{l}150.0 \\
112.0\end{array}$ & $\begin{array}{l}160.0 \\
110.0\end{array}$ \\
\hline
\end{tabular}

"The fourteen FWR assemblies label the rows and are grouped by burnup and cooling time. The column labeled "l" shows the overhead detector readings when each assembly was isolated. Except for experimental uncertainties, these should be the results of the unfolding operations. The other column labels show the number of assemblies in the contrived arrays.

Columns $2 a$ and $2 c$ had two assemblies separated by a void; Jlumns $2 b$ and $2 d$ had two adjacent assemblies.

Columns $3 \mathrm{a}$ and $3 \mathrm{~b}$ had a linear array of three adjacent assemblies with a strong activity assenbly on one end, a medium activity assembly on the other end, and a medium or weak assembly in the middle.

The $6 \mathrm{a}$ and $6 \mathrm{~b}$ arrays were both triangular with different sets of assemblies present.

The rarger arrays were within a 4 by 4 square. 


\section{The Calculated of Source Activities}

While unfolding the $d$ 's in the various arrays, several sets of Cs were employed. If the data in Fig. 5 are used literally, the unfolded d's are clearly inferior to those in column "l" of Table I, especially for the weaker sources. Using Fig. 5 as merely a guide and smoothing each set of Cs gave somewhat better results.

The best results, those in the rest of Table I, followed from using the solid curve in Fig. $6(b)$. All the assemblies, including those with the lowest burnup, were given the same $C s: 1.00,0.20,0.05$, and 0.02 as the detector moved further away. The first three values are for the locations shown in Fig. 2(b); the last value is for a location one more position away from the side of the assembly. (The array resembles an upside down "T".) No negative source activities were obtained for some of the weak sources (unlike previous attempts with other $(\mathrm{CS})$ and the overall comparison with the measured d's was the best found. More than half the values in Table I differ by less than 5 percent from the measured d's, 70 percent of them differ by less than 10 percent, and 88 percent differ by less than 20 percent. The accuracy increases with burnup.

Using only the three largest Cs (1.00, 0.20, and 0.05) gave solutions slightly larger and less accurate than those in Table I.

For these PWR assemblies, a single set of $C s$ is not only convenient but accurate to use. This means that an inspector would not have to isolate and measure the Cs for assemblies from different core loadings, and the input. to the computer code is simplified.

\section{Void Detection}

To examine further the accuracy of the unfolding process for this PWR exercise, the 4 by 4 arrays with voids were redone, but the voids were not ignored. Each void was assigned an "assembly" of zero source activity and all of its Cs were zero. After unfolding was accomplished, the computed d's in a total of 17 voids in five separate arrays ranged from -0.2 to 11.0 instead of the ideal zero. The average value for the individual voild position was about 6. Since the three assemblies with the lowest burnups had values in this range, it follows that an inspector could not detect an assembly with very low burnup from a dumy assembly using this technique, if there are neighboring assemblies of much higher activity. 
The same conclusion is reaciied from measurements taken above each member of a 10 by 16 array of assemblies in the storage pond. Values above assemblies with the same burnup and cooling time were very dependent on the activities of the neighbors.

\section{v. CONCLUSIONS}

The unfolding of individual source activities from data taken above an array of spent-fuel assemblies with an uncollimated detector can be very important in determining the actual activities within the array. With a variety of source strengths dispersed throughout the array, a single detector response can be practically meaningless when neighboring assemblies also contribute to that response. Even the detection of a void is not certain under these conditions.

An exercise with as many as 14 PWR assemblies has shown that the unfolding can be performed with an accuracy adequate for IAEA inspection purposes when the individual source strengths in the array do not differ by factors of 5 or more. But weak source strengths were not reliably found when unfolded along with much stronger sources. In fact, they could not be distinguished from voids.

Studies with simulated larger arrays indicate that the small unavoidable fluctuations in measured values are likely to lead to much larger adverse cumulative effects in the unfolded results.

This study has thus shown only limited uses for the unfolding of data from an uncollimated detector. The defects are the inability to accurately recover weak source strengths when among stronger sources and the apparently rapid loss of accuracy as the number of assemblies in an array increases.

\section{ACKNOWLEDGMENTS}

Howard Menlove and Q-5 group created the opportunity to undertake this study. The PWR data was provided by David Lee, John Phillips, and Jim Halbig. Gene Bosler's information about the Central Computing Facility was most helpful. All of this assistance is greatly appreciated. The support of the Internationl Safeguards Project Office and the Office of Safeguards and Security is gratefully acknowledged. 


\section{DEDUCTIONS FROM A SIMPLE DETECTOR'S MEASUREMENT}

\section{BURNUP AND REACTOR POWER HISTORY}

The burnup of an assembly is usually given in megawatt-days per metric ton of initiai uranium, but it is more convenient here to express this in terms of the fraction of heavy nuclides that could and did fission. A somewhat simplified expression for burnup is used to give an indication of the nature of the situation only.

$$
B U(M N d / t U)=46.977 E \frac{\sum_{i} n_{i} \sigma_{i}}{\sum_{i} n_{i}} .
$$

A fissionable nuclide here has a number density $n_{i}$ and a fission cross section $\sigma_{i}$; the average energy release per fission (in MeV) is $E$. The neutron fluence (neutrons $/ \mathrm{cm}^{2}$ ) is $\sigma \tau$, the flux times the exposure.

The reactor's power history is also connected to the fluence, as seen by examining the total number of fissions that occurred during a constant power $P$ portion of the history.

$$
\frac{P}{E}=V \quad \sigma \tau \sum_{i} n_{i} \sigma_{i}
$$

The average fluence is taken over the reactor's volume $v$.

$$
\phi \tau=\frac{P}{E \sum_{i} n_{i} \sigma_{i}} .
$$

A realistic power history would have a set of these fluence-power relations. 
Before finding the effect of an irradiated assembly on a detector, its emissions must be known. For gamina rays as an example, the work of Ref. 5 is used. The character and amount of emission depends on three parameters already introduced: $\phi, \tau$, and $t$. A specific situation is shown in Fig. A-1, based on results in Ref. 6 . Gamma rays with energies below $1 \mathrm{MeV}$ dominate, so only these are usad in the following calculation.

For an exploratory analysis, the dose rate in a gross gamma detector is taken to be this sum over the gamma-ray energies:

$$
D(\phi, \tau, t)=\sum_{E} \mu_{a}(E) E N(E, \phi, \tau, t),
$$

where the number rate $N$ is taken from Ref. 6 and $\mu_{a}$ is the absorption coefficient for the detector. Any attenuation or buildup by a surrounding medium is ignored in this approximate analysis.

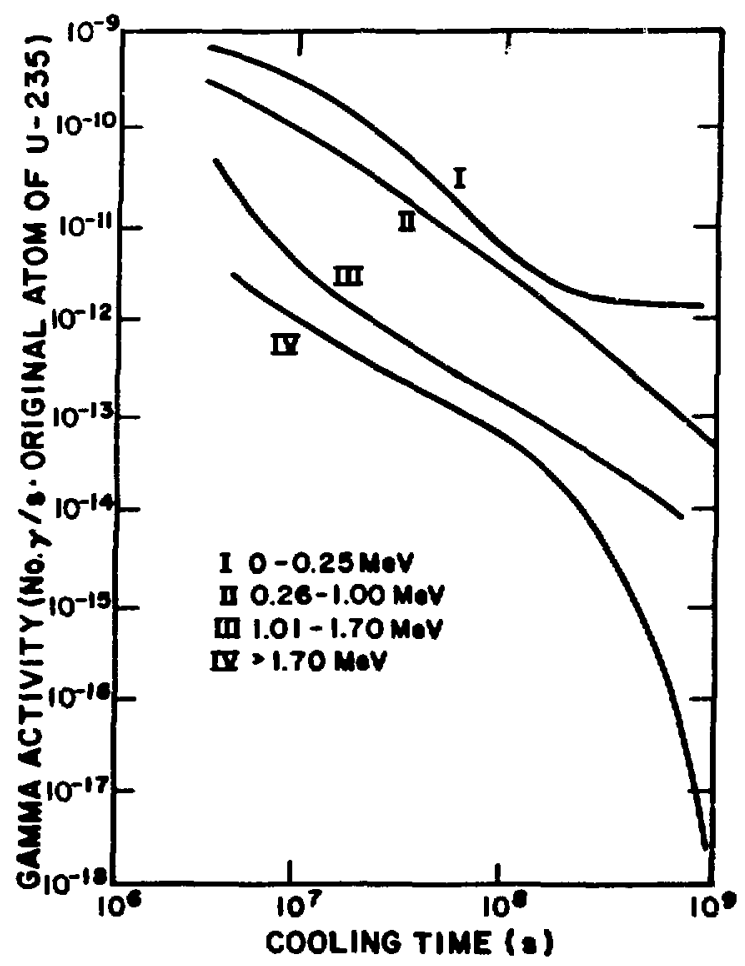

Fig. A-1.

The gamma activity from an irradiated assembly from Ref. 5 is shown here, split into four energy ranges. The two lower ranges (I and II) dominate the activity for the cooling times shown. 
For a simple $\mathrm{UO}_{2}(2.5$ percent enriched) fuel, Eq. (A-1) gives

$$
\phi \tau=7.6 \times 10^{6} \mathrm{BU}(\mathrm{MWd} / \mathrm{tU}) \text {. }
$$

By specifying the burnup and flux, the dose rate versus cooling time curves in Fig. A-2 are found. Of particular interest is the crossing of some of the curves. This illustrates the obvious fact that a dose rate in the gross-gamma detector does not uniquely specify burnup and cooling time. The measurements can be used as a consistency check on the specified values, however. With confidence in the power history and cooling-time parameters, a burnup could be deduced.

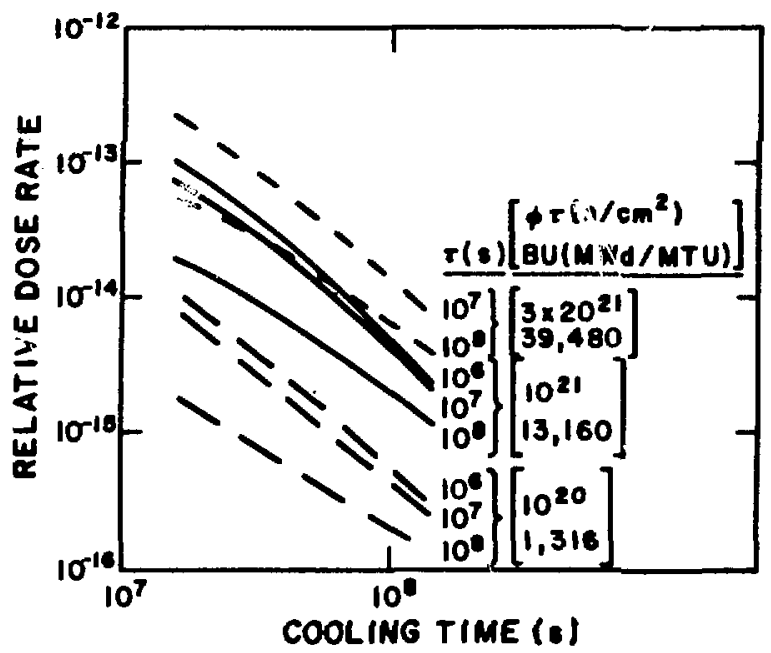

Fig. A-2.

Relative values of dose rates in an air-filled chamber are shown for several combinations of burnup, irradiation time, and cooling time.

\section{APPENDIX B}

\section{LINEARIZATION PROCEDURE FOR A NONLINEAR DETECTOR RESPONSE}

Let $D_{x_{0} y_{0}}$ be the dose rate in a nonlinear detector above the $\left(x_{0}, y_{0}\right)$
location. The detector's response $R_{x_{0} y_{0}}$ is some arbitrary, but krown, function $f$ of the dose rate.

$$
R_{x_{0} y_{0}}=f\left(D_{x_{0} y_{0}}\right)
$$


Since the $d_{x y}$ in Eq. (1) are the u...nnowns and the $D_{x_{0} y_{0}}$ depend on them, the $R_{x_{0} v_{0}}$ are written as a function of all of them

$$
R_{x_{0} y_{0}}=f\left(\left\{d_{x y}\right\}\right)
$$

On the right-hand side it is understood that the detector is located at $\left(x_{0}, y_{0}\right)$. This is a set of nonlinear equations for the $d_{x y^{*}}$ The $R_{x_{0} y_{0}}$ are measured and the $C_{x y} x_{0}$ are assumed known.

Equation (B-2) can be linearized by truncating a Taylor series expansion about an initial estimated $d_{x y}^{0}$.

$$
R_{x_{0} y_{0}}=f\left(\left\{d_{x_{0} y_{0}}^{o}\right\}\right)+\sum_{x} \sum_{y} \frac{\delta f\left(\left\{d_{x y}\right\}\right)}{\delta d_{x y}} \mid\left\{d_{x y}^{o}\right\} \Delta d_{x y} .
$$

The partial derivative is simplified to the following form:

$$
\frac{\delta f\left(\left\{d_{x y}\right\}\right)}{\delta d_{x y}}=\frac{\delta f\left(D_{x_{0} y_{0}}\right)}{\delta D_{x y}}=\frac{\delta f\left(D_{x_{0} y_{0}}\right)}{\delta D_{x_{0} y_{0}}} \frac{\delta D_{x_{0} y_{0}}}{\delta d_{x y}}=C_{x y}^{x_{0} y_{0}} \frac{\delta f\left(D_{x_{0} y_{0}}\right)}{\delta D_{x_{0} y_{0}}} .
$$

If $f(s)=a s^{2}+b s$, for example, then Eq. (B-4) gives $\left(2 a D_{x_{0}} y_{0}+b\right) c_{x_{0}}^{x_{0} y_{0}}$. Whatever the functional form, Eq. (B-3) is a set of Pinear equations for the $\Delta d_{x y}$. They can be solved and the results used to improve the estimate to $d_{x y}^{0 x y}+\Delta d_{x y}$. If these new $d_{x y}$ values do not satisfy Eq. (B-2) accurately enough, they become the $d_{x y}^{0}$ in Eq. (B-3) and another set of changes in $d_{x y}$ are calculated. This iteration process continues until the desired accuracy is finally achieved. 


\section{REFERENCES}

1. "The Structure and Content of Agreements Between the Agency and States Required in Connection with the Treaty on the Non-Proliferation of Nuclear Weapons," International Atomic Energy Agency report INFCIRC/153 (corrected) (June 1972).

2. M. L. James, C. M. Smith, and J. C. Wolford, Applied Numerical Methods for Digital Computation (Harper and Row, New York, 1971), 2nd Edition, Chap. 3.

3. S. S. Kuo, Computer Applications of Numerical Methods (Addison-Wesley, Reading, MA, 1972), Chap. 8.

4. C. E. Moss and D. M. Lee, "Gross Gamma-Ray Measurements of LWR Spent Fuel Assemblies in Underwater Storage Arrays," Los Alamos Scientific Laboratory report LA-8447 (to be published).

5. P. M. Rinard, "Gamma Doses From Irradiated Assemblies Under Water," Los Alamos Scientific Labc'atory report LA-7914-MS (July 1979).

6. J. 0. Blomeke and M. F. Todd, "IIranium-235 Fission Product Production as a Function of Thermal Neutron Five, Irradiation Time, and Decay Time, I. Atomic Concentrations and Gross Totals," Oak, Ridge National Laboratory report ORNL-2127, Part I, Vol. 2 (August 19, 1957). 